\title{
Association Between Blood PLT and RBC Related Indices and Disease Activity in Patients with Rheumatoid Arthritis
}

\author{
Li Xue $\mathbb{D}^{\prime}$ \\ Li Tao' \\ Haifeng Sun ${ }^{2}$ \\ Yan Wang' \\ Yanping Zhang' \\ $\mathrm{Na}$ Lei $^{1}$ \\ Zeshi Liu' \\ Hua Zhang' \\ Li Jin' \\ Ting Zhang' \\ Jing Zhang' \\ Hao Meng' \\ Fang Huang' \\ Yan Geng' \\ Ming $\mathrm{Li}^{3}$ \\ 'Department of Clinical Laboratory, The \\ Second Affiliated Hospital of Xi'an \\ Jiaotong University, Xi'an, 710004, \\ People's Republic of China; ${ }^{2}$ Third \\ Department of Medical Oncology, \\ Shaanxi Provincial Cancer Hospital \\ Affiliated to Medical College of Xi'an \\ Jiaotong University, Xi'an, 7I006I, \\ People's Republic of China; ${ }^{3}$ Department \\ of Cardiovascular Surgery, The First \\ Affiliated Hospital of Xi'an Jiaotong \\ University, Xi'an, 71006I, People's \\ Republic of China
}

Correspondence: Yan Geng Department of Clinical Laboratory, The Second Affiliated Hospital of Medical College of Xi'an Jiaotong University, Xi'an, People's Republic of China

Email gengyanjyk@126.com

Ming Li

Department of Cardiovascular Surgery,

The First Affiliated Hospital of Xi'an

Jiaotong University, Xi'an, People's

Republic of China

Email minglil@।26.com
Background: Platelet (PLT) and red blood cell (RBC) have been demonstrated to play a critical role in inflammatory processes. This study aimed to evaluate the association of blood PLT and RBC related parameters with the disease activity in rheumatoid arthritis (RA) patients, and also to investigate the role of these indices in differentiating among RA patients with different disease activity.

Methods: Clinical data from RA patients were retrospectively analyzed. RA patients were divided into inactive group and active group according to DAS28-CRP. The relationship between blood PLT and RBC counts-related indices and DAS28-CRP was detected by Spearman correlation. ROC curve was used to assess the diagnostic value of these indices in differentiating active RA from inactive RA.

Results: Active RA patients exhibited higher level of PLT counts but significantly lower levels of RBC counts, hemoglobin ( $\mathrm{Hb}$ ), red blood cells-platelet ratio (RPR) and hemoglobin-platelet ratio (HPR) compared with inactive RA. PLT counts were positively but RBC counts, Hb, RPR and HPR were negatively related with DAS28-CRP.

Conclusion: Blood PLT and RBC related indices were significantly associated with RA disease activity. These indices may be used to distinguish active RA from inactive RA.

Keywords: rheumatoid arthritis, platelets, red blood cells, hemoglobin

\section{Introduction}

Rheumatoid arthritis (RA) is a chronic autoimmune disease characterized by synovial hyperplasia, pannus formation, and progressive damage of articular cartilage and bone. ${ }^{1}$ RA patients are challenged to physical disabilities and a significant economic burden with disease progression. Previous studies revealed that early diagnosis and treatment could prevent bone destruction and joint deformities caused by RA, and thus contribute to a greater rate of remission in RA patient. ${ }^{2}$ Therefore, $^{2}$ early diagnosis and treatment are vital in improving the prognosis of RA.

Currently, the 2010 American College of Rheumatology (ACR) and European League Against Rheumatism (EULAR) classification criteria for RA has been widely used for diagnosis in clinical practice. The 2010 ACR/EULAR RA classification criteria place more emphasis on circulating anti-cyclic citrullinated peptide (anti-CCP), rheumatoid factor (RF) and other serological biomarkers, in comparison with the 1987 ARA classification criteria. ${ }^{3}$ It was reported that CCP rules are not useful in identifying a proportion of early unclassified RA patients. ${ }^{4}$ Both antiCCP and RF are members of the autoantibodies family. Anti-CCP has high 
specificity but relatively low sensitivity for RA, while RF is strongly sensitive but lacks specificity for RA patients. ${ }^{5}$ Therefore, there is a need for establishing new serological biomarkers to realize the early diagnosis of RA.

Recently accelerating studies have demonstrated the critical role of platelet (PLT) in inflammatory processes. For instance, PLT could participate in the regulation of leukocyte recruitment by releasing numerous inflammatory mediators. ${ }^{6}$ P-selectin and several adhesion molecules expressed on PLT could contribute to the interaction between platelets and other leukocytes such as neutrophils, monocytes, $\mathrm{T}$ cells and so on. ${ }^{7,8}$ Recent data indicate that anemia could occur in the setting of autoimmune diseases in that bone marrow function and iron metabolism could be influenced by inflammation. ${ }^{9}$ It is reported that inflammatory cytokines may suppress maturation of red blood cell (RBC). ${ }^{10,11}$ Therefore, it has been suggested that RBC-related parameters such as red blood cell distribution width (RDW) and hemoglobin ( $\mathrm{Hb}$ ) contents may be used as inflammatory indicators for predicting the severity of several autoimmune diseases including systemic lupus erythematosus, ${ }^{12,13}$ primary Sjögren's syndrome, ${ }^{14}$ and autoimmune hepatitis. ${ }^{15}$ However, there are few studies that have systematically assessed the association of PLT, $\mathrm{RBC}, \mathrm{Hb}$, red blood cells-platelet ratio (RPR) and hemoglobin-platelet ratio (HPR) with the disease activity of RA patients. And little is known about the diagnostic value of the peripheral blood PLT and RBC related indices in distinguishing between active RA and inactive RA. Therefore, this study aimed to investigate the correlation of hematological indices including PLT, RBC, Hb, RPR and HPR with RA disease activity. We also evaluated the diagnostic role of these indices in differentiating among RA patients with different disease activity.

\section{Materials and Methods}

\section{Study Population}

This study included one hundred and seventy-eight RA patients who were admitted to the Department of Rheumatology and Immunology of the Second Affiliated Hospital of Xi'an Jiaotong University during the period from September 2017 to April 2020. Patients fulfilled the 2010 ACR/EULAR criteria for RA. ${ }^{16}$ Patients who had hematologic diseases, other autoimmune inflammatory diseases, infections, malignancies, or had any history of other chronic diseases such as diabetes mellitus, dyslipidemia, thyroid dysfunction, severe liver or kidney impairment as well as those receiving treatment with corticosteroids within the last 3 months were excluded. One hundred and sixty-four healthy individuals were recruited from the health examination center of the same hospital, and matched with RA patients for age and gender. The study was conducted in accordance with the declaration of Helsinki and approved by the Research Committee of Human Investigation of Xi'an Jiaotong University Health Science Center and the written informed consent was given by all subjects. All methods were performed in accordance with the relevant guidelines and regulations.

\section{Assessment of Disease Activity}

Using the Disease Activity Score in 28 joints based on C-reactive protein (DAS 28-CRP), ${ }^{17,18}$ disease activity of RA patients can be described as low (DAS 28 -CRP $\leq 2.7$ ), moderate $(2.7<$ DAS 28 -CRP $\leq 4.1)$ or high (DAS 28 CRP $>4.1$ ), respectively. We define patients with moderate and high disease activity as active RA, whereas those with low disease activity were defined as inactive RA.

\section{Clinical and Laboratory Parameters}

Patients' characteristics, including age, gender, medical history, symptoms and signs, diagnosis, treatment, laboratory testing results were gathered from their electronic medical records. The laboratory testing results included PLT, platelet distribution width (PDW), white blood cells (WBC), lymphocytes, neutrophils, monocytes, RBC, $\mathrm{Hb}$, red blood cell-specific volume (HCT), RDW, erythrocyte sedimentation rate (ESR), C-reactive protein (CRP), RF, anti-CCP. RPR and HPR were calculated.

\section{Statistical Analysis}

Continuous variables with the normal distribution were presented as mean values \pm standard deviation. Nonnormally distributed data were presented as median (interquartile range). Categorical variables were expressed as frequencies or percentages. The differences of continuous variables were compared by The Student's $t$-test or MannWhitney $U$-test, while the chi-square test was performed to compare the differences of categorical variables. Spearman correlation analysis was used to detect the association between variables. Receiver operating characteristic (ROC) curves were plotted to distinguish RA patients from healthy individuals or to differentiate active RA from inactive group. The area under the curve (AUC) and $95 \%$ confidence interval (CI) were calculated to evaluate the diagnostic value of each indice. The optimal cut- 
Table I Comparison of Clinical Characteristics Between RA Patients and Controls

\begin{tabular}{|c|c|c|c|}
\hline & RA $(\mathbf{N}=\mid 78)$ & Control $(N=164)$ & $P$-value \\
\hline Age (years) & $57.11 \pm 14.17$ & $54.86 \pm 10.59$ & 0.122 \\
\hline Gender (F/M) & $132 / 46$ & $126 / 38$ & 0.616 \\
\hline BMI $\left(\mathrm{kg} / \mathrm{m}^{2}\right)$ & $25.6 \pm 3.1$ & $26.1 \pm 3.3$ & 0.376 \\
\hline CRP (mg/L) & $18.35(6.75-52.4 \mid)$ & $2.1(1.1-3.2)$ & $<0.001$ \\
\hline $\operatorname{ESR}(\mathrm{mm} / \mathrm{h})$ & $37.0(15-79)$ & $5.22(2.73-12.81)$ & $<0.001$ \\
\hline $\mathrm{RF}(\mathrm{IU} / \mathrm{mL})$ & $|67.3|(23.2-426.00)$ & $4.87(3.10-15.70)$ & $<0.001$ \\
\hline Anti-CCP $(\mathrm{U} / \mathrm{mL})$ & $246.3(38.1-400.0)$ & |3.2(5.7-19.3) & $<0.001$ \\
\hline WBC $\left(10^{9} / \mathrm{L}\right)$ & $6.21 \pm 2.77$ & $6.19 \pm 2.54$ & 0.955 \\
\hline $\operatorname{RBC}\left(10^{12} / \mathrm{L}\right)$ & $4.06 \pm 0.93$ & $4.62 \pm 0.74$ & $<0.001$ \\
\hline $\mathrm{Hb}(\mathrm{g} / \mathrm{L})$ & $115.20 \pm 28.08$ & $136.18 \pm 2 \mid .67$ & $<0.001$ \\
\hline HCT (\%) & $35.45 \pm 8.59$ & $41.03 \pm 5.84$ & $<0.001$ \\
\hline RDW & $14.16 \pm 4.42$ & $13.45 \pm 2.42$ & 0.075 \\
\hline $\operatorname{PLT}\left(10^{9} / \mathrm{L}\right)$ & $240.59 \pm 73.28$ & $205.05 \pm 53.05$ & $<0.001$ \\
\hline PDW & $|2.42 \pm 4.4|$ & $12.97 \pm 3.90$ & 0.263 \\
\hline RPR & $0.019 \pm 0.0065$ & $0.024 \pm 0.0072$ & $<0.001$ \\
\hline HPR & $0.55 \pm 0.21$ & $0.727 \pm 0.23$ & $<0.001$ \\
\hline
\end{tabular}

Abbreviations: F, female; M, male; BMI, body mass index; CRP, C-reactive protein; ESR, erythrocyte sedimentation rate; RF, rheumatoid factor; Anti-CCP, anti-cyclic citrullinated peptide; WBC, white blood cells; RBC, red blood cells; Hb, hemoglobin; HCT, red blood cell specific volume; RDW, red blood cell volume distribution width; PLT, platelet; PDW, platelet volume distribution width; RPR, red blood cells-platelet ratio; HPR, hemoglobin-platelet ratio.

off value, sensitivity, specificity, positive predictive value (PPV), negative predictive value (NPV) and accuracy (AC) of the indices were assessed.

\section{Results}

\section{Study Population}

Clinical and laboratory characteristics of RA patients and healthy controls were shown in Table 1 . There were no differences in age and gender distribution, body mass index (BMI) and $\mathrm{WBC}$ between the two groups. Besides, CRP, ESR, RF and anti-CCP in RA patients were significantly higher than those in the control group $(P<0.001)$. Table 2 showed the clinical and laboratory characteristics of two groups of RA patients. Age, gender, BMI, disease duration, anti-CCP and WBC were similar between the two groups. The active RA patients had higher levels of ESR, CRP and RF compared with inactive RA groups $(P<$ 0.001 or $P<0.01)$. The greater levels of Ig G, Ig A and Ig $M$ were also observed in active RA groups as compared to those in inactive RA groups $(P<0.05)$.

\section{Blood Counts of PLT, RBC and Other Related Parameters}

As shown in Table 1, blood counts of PLT were elevated while RBC as well as levels of Hb, HCT, RPR and HPR were decreased in RA patients compared with healthy controls $(P<0.001)$. There were no significant differences in PDW and RDW between the two groups $(P>0.05)$.

Patients with active RA showed higher levels of PLT counts but lower levels of RBC counts, HCT, RPR and HPR than those with inactive group $(P<0.001$ or $P<$ 0.01 , Table 2). No significant differences in PDW and RDW were observed between the active RA and inactive RA $(P>0.05$, Table 2).

In order to evaluate the diagnostic performance of blood PLT, RBC, Hb, RPR and HPR, we further used ROC curve analysis. As shown in Table 3, the AUC of PLT, RBC, Hb, RPR and HPR was 0.648, 0.785, 0.787, 0.747 and 0.738 respectively (all $P<0.001$ )for RA patients versus healthy controls. There were significant differences in the AUC between PLT and RBC as well as between $\mathrm{Hb}$ and PLT for distinguishing RA patients from healthy controls $(P<0.05$ or $P<0.01$, Figure 1$)$. And no significant difference was observed in the AUC between $\mathrm{RBC}$ and $\mathrm{Hb}$ for distinguishing RA patients from healthy controls $(P>0.05$, Figure 1).

Table 4 showed the ROC curve analysis for the active and inactive RA patients. The AUC of PLT, RBC, Hb, RPR and HPR was 0.666, 0.780, 0.786, 0.744 and 0.727 for active RA versus inactive RA (all $P<0.001$ ). The optimal cutoff values of PLT, RBC, Hb, RPR and HPR between active RA and inactive RA groups were $243 \times 10^{9}$ / $\mathrm{L}, 4.22 \times 10^{12} / \mathrm{L}, 121 \mathrm{~g} / \mathrm{L}, 0.48$ and 0.017 . As shown in 
Table 2 Comparison of Clinical Characteristics Between the Two RA Subgroups

\begin{tabular}{|c|c|c|c|}
\hline & Inactive RA ( $\mathbf{N}=\mathbf{8 8})$ & Active RA ( $\mathrm{N}=90)$ & $P$-value \\
\hline Age (years) & $56.29 \pm 15.16$ & $58.41 \pm 13.33$ & 0.121 \\
\hline Gender (F/M) & $67 / 21$ & $68 / 22$ & 0.928 \\
\hline BMI $\left(\mathrm{kg} / \mathrm{m}^{2}\right)$ & $25.5 \pm 3.1$ & $24.7 \pm 2.3$ & 0.325 \\
\hline Disease duration (years) & $7.2 \pm 3.8$ & $8.6 \pm 5.1$ & 0.213 \\
\hline CRP (mg/L) & $9.65(4.92-18.35)$ & $32.07(9.13-55.72)$ & $<0.001$ \\
\hline $\operatorname{ESR}(\mathrm{mm} / \mathrm{h})$ & $30.0(14-65)$ & $54.0(19-87)$ & $<0.001$ \\
\hline $\mathrm{RF}(\mathrm{IU} / \mathrm{mL})$ & $\mid 29.05(20.80-32 \mid .00)$ & $261.29(32.56-910.00)$ & $<0.01$ \\
\hline Anti-CCP $(\mathrm{U} / \mathrm{mL})$ & $255.7(21.1-382.0)$ & $302.2(88.3-500.0)$ & 0.264 \\
\hline $\lg G(g / L)$ & $\mid 1.97 \pm 3.21$ & $15.42 \pm 6.35$ & $<0.05$ \\
\hline $\lg A(g / L)$ & $2.36 \pm 1.37$ & $3.19 \pm 1.93$ & $<0.05$ \\
\hline $\lg M(g / L)$ & $1.05 \pm 0.78$ & $|.37 \pm 0.9|$ & $<0.05$ \\
\hline WBC $\left(10^{9} / \mathrm{L}\right)$ & $6.22 \pm 2.24$ & $6.64 \pm 2.74$ & 0.270 \\
\hline $\mathrm{RBC}\left(10^{12} / \mathrm{L}\right)$ & $4.37 \pm 0.50$ & $3.87 \pm 0.58$ & $<0.001$ \\
\hline $\mathrm{Hb}(\mathrm{g} / \mathrm{L})$ & $127.83 \pm 17.55$ & $109.08 \pm 16.10$ & $<0.001$ \\
\hline НCT (\%) & $38.18 \pm 6.74$ & $35.64 \pm 5.22$ & $<0.01$ \\
\hline RDW & $|4.40 \pm 4.1|$ & $14.66 \pm 2.54$ & 0.662 \\
\hline $\operatorname{PLT}\left(10^{9} / \mathrm{L}\right)$ & $215.61 \pm 60.95$ & $257.00 \pm 76.27$ & $<0.001$ \\
\hline PDW & $12.74 \pm 3.56$ & $12.92 \pm 3.98$ & 0.754 \\
\hline RPR & $0.022 \pm 0.0068$ & $0.017 \pm 0.0055$ & $<0.001$ \\
\hline HPR & $0.64 \pm 0.22$ & $0.48 \pm 0.18$ & $<0.001$ \\
\hline
\end{tabular}

Notes: Patients in inactive group with a DAS 28-CRP score lower than 2.7; active patients with a DAS 28-CRP score of 2.7 and higher.

Abbreviations: DAS28-CRP, Disease Activity Score in 28 joints based on C-reactive protein; F, female; M, male; BMI, body mass index; CRP, C-reactive protein; ESR, erythrocyte sedimentation rate; RF, rheumatoid factor; Anti-CCP, anti-cyclic citrullinated peptide; Ig G, immunoglobulin G; Ig A, immunoglobulin A; Ig M, immunoglobulin M; WBC, white blood cells; RBC, red blood cells; Hb, hemoglobin; HCT, red blood cell specific volume; RDW, red blood cell volume distribution width; PLT, platelet; PDW, platelet volume distribution width; RPR, red blood cells-platelet ratio; HPR, hemoglobin-platelet ratio.

Table 3 The Diagnostic Value of PLT, RBC, Hb, RPR and HPR for RA

\begin{tabular}{|l|l|l|l|l|l|l|l|l|}
\hline Parameters & AUC & $\mathbf{9 5 \%}$ Cl & Optimal Cut-off Value & Specificity (\%) & Sensitivity (\%) & PPV (\%) & NPV (\%) & AC (\%) \\
\hline PLT(I0\%/L) & 0.648 & $0.584-0.709$ & $>237$ & 82.26 & 46.55 & 72.41 & 60.61 & 64.41 \\
RBC (I0'/2) & 0.785 & $0.727-0.835$ & $<4.29$ & 77.42 & 72.63 & 76.28 & 73.88 & 75.03 \\
Hb (g/L) & 0.787 & $0.730-0.837$ & $<128$ & 77.42 & 73.74 & 76.56 & 74.67 & 75.58 \\
RPR & 0.747 & $0.687-0.801$ & $<0.018$ & 90.32 & 55.17 & 85.07 & 66.83 & 72.75 \\
HPR & 0.738 & $0.677-0.793$ & $<0.54$ & 83.87 & 55.75 & 77.56 & 65.46 & 69.81 \\
\hline
\end{tabular}

Abbreviations: AUC, area under curve; $95 \%$ Cl, 95\% confidence interval; PPV, positive predictive value; NPV, negative predictive value; AC, accuracy; RBC, red blood cells; $\mathrm{Hb}$, hemoglobin; PLT, platelet; RPR, red blood cells-platelet ratio; HPR, hemoglobin-platelet ratio.

Figure 2, $\mathrm{RBC}$ and $\mathrm{Hb}$ had a higher AUC relative to ESR, $\mathrm{RF}$ and anti-CCP in distinguishing active RA from inactive RA $(P<0.01$ or $P<0.001)$. There was no significant difference in the AUC between PLT and other indices including ESR, CRP, RF and anti-CCP for distinguishing active RA patients from inactive group $(P>0.05)$.

\section{Correlation of Blood PLT and RBC} Related Indices with Disease Activity and Laboratory Parameters

To further evaluate the relationship between blood PLT and $\mathrm{RBC}$ related indices and disease activity, we conducted a correlation analysis between blood levels of these indices and disease activity index DAS-28 CRP and laboratory parameters including CRP and ESR.

As shown in Table 5, PLT was positively associated with DAS-28 CRP ( $\mathrm{r}=0.327, P<0.001)$, CPR ( $\mathrm{r}=0.284$, $P<0.001)$ and ESR $(\mathrm{r}=0.331, P<0.001)$. RBC was negatively associated with DAS-28 CRP ( $\mathrm{r}=-0.428, P<$ $0.001)$, CRP ( $\mathrm{r}=-0.289, P<0.001)$ and ESR $(\mathrm{r}=-0.481$, $P<0.001)$. Hb was negatively associated with DAS-28 CRP (r $=-0.489, P<0.001)$, CRP $(\mathrm{r}=-0.341, P<0.001)$ and $\operatorname{ESR}(\mathrm{r}=-0.569, \mathrm{P}<0.001)$. RPR was negatively associated with DAS-28 CRP ( $\mathrm{r}=-0.310, P<0.001)$, CRP (r $=-0.397, P<0.001)$ and ESR $(\mathrm{r}=-0.329, P<$ 

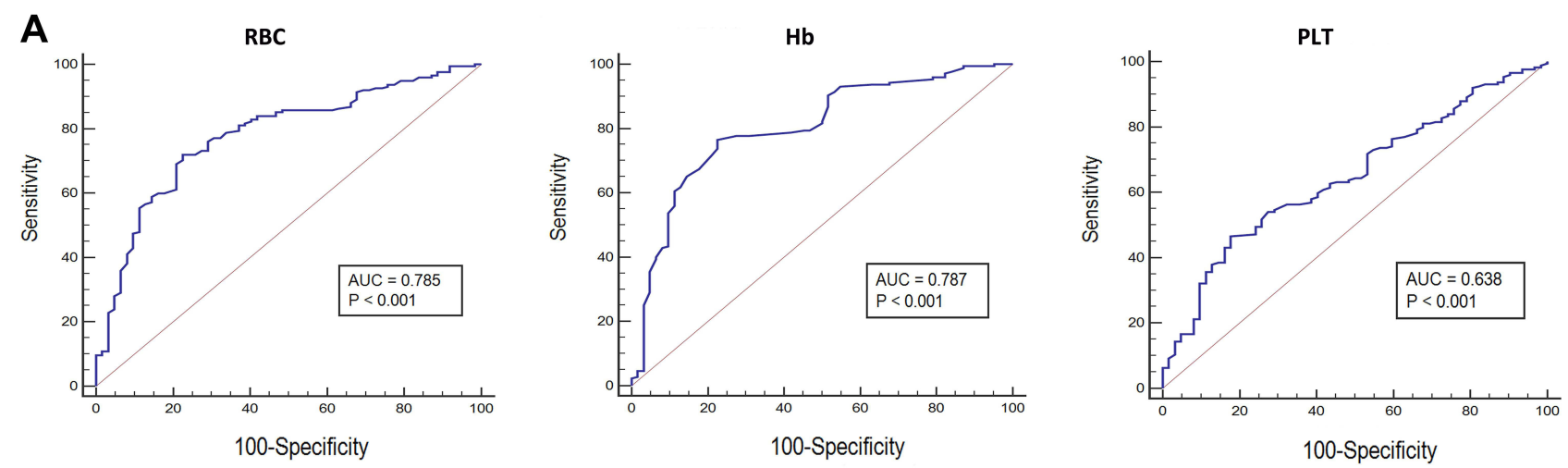

B
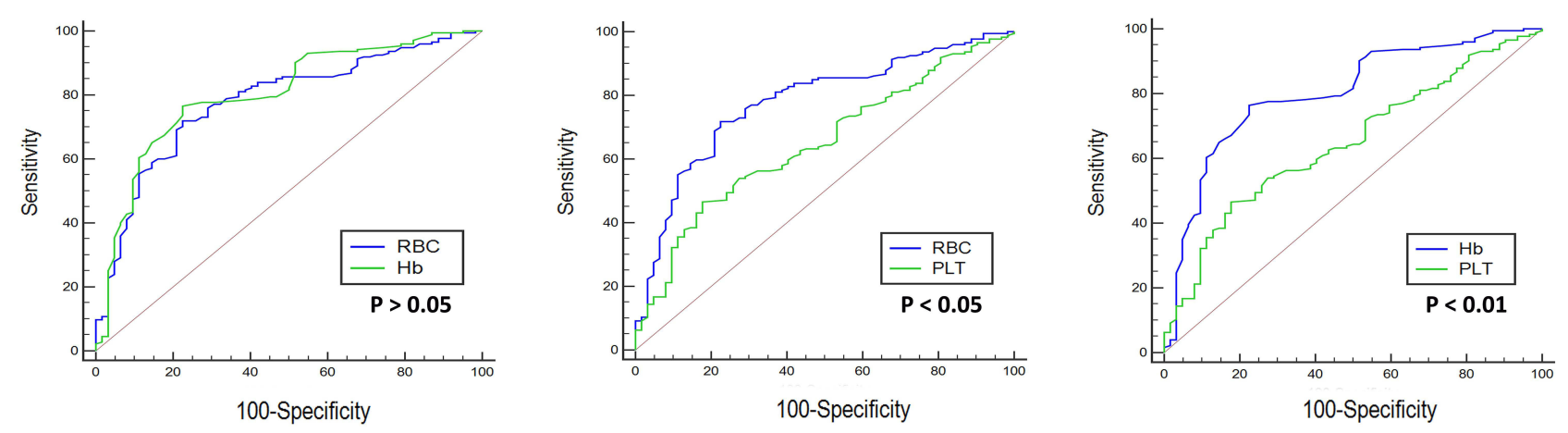

Figure I Performance of blood RBC, Hb, and PLT in discriminating RA patients from healthy controls. (A) The ROC curves of the three indices in differentiating RA patients from healthy controls. (B) Comparison of ROC curves among the three indices.

Abbreviations: ROC, receiver operating characteristic curve; AUC, area under the curve; RBC, red blood cells; Hb, hemoglobin; PLT, platelet.

0.001). HPR was negatively associated with DAS-28 CRP $(\mathrm{r}=-0.293, P<0.001)$, CRP $(\mathrm{r}=-0.402, P<0.001)$ and $\operatorname{ESR}(\mathrm{r}=-0.362, P<0.001)$.

Notably, the correlation between $\mathrm{Hb}$ and disease activity parameters such as ESR and DAS-28 CRP was the strongest.

\section{Discussion}

In this retrospective study of one hundred and seventyeight patients, we systematically analyzed the association of PLT, RBC, Hb, RPR and HPR with the disease activity in a cohort of Chinese patients with RA. We focused on the association of these indices with the current disease

Table 4 The Diagnostic Value of the Markers to Distinguish Active RA from Inactive RA

\begin{tabular}{|l|l|l|l|l|l|l|l|l|}
\hline Parameters & AUC & $\mathbf{9 5 \%} \mathbf{C l}$ & $\begin{array}{l}\text { Optimal Cut-off } \\
\text { Value }\end{array}$ & $\begin{array}{l}\text { Specificity } \\
\text { (\%) }\end{array}$ & $\begin{array}{l}\text { Sensitivity } \\
\text { (\%) }\end{array}$ & $\begin{array}{l}\text { PPV } \\
\text { (\%) }\end{array}$ & $\begin{array}{l}\text { NPV } \\
\text { (\%) }\end{array}$ & $\begin{array}{l}\text { AC } \\
\text { (\%) }\end{array}$ \\
\hline PLT $\left(I 0^{9} / \mathrm{L}\right)$ & 0.666 & $0.591-0.736$ & $>243$ & 73.91 & 56.19 & 68.29 & 62.78 & 65.05 \\
RBC $\left(I 0^{12} / \mathrm{L}\right)$ & 0.780 & $0.711-0.839$ & $<4.22$ & 68.12 & 83.96 & 72.48 & 80.94 & 76.04 \\
Hb $(\mathrm{g} / \mathrm{L})$ & 0.786 & $0.717-0.844$ & $<121$ & 68.12 & 81.13 & 71.79 & 78.31 & 74.63 \\
RPR & 0.744 & $0.673-0.807$ & $<0.017$ & 68.12 & 74.29 & 69.97 & 72.60 & 71.21 \\
HPR & 0.727 & $0.654-0.791$ & $<0.48$ & 81.16 & 56.19 & 74.89 & 64.94 & 68.68 \\
CRP $(\mathrm{mg} / \mathrm{L})$ & 0.749 & $0.678-0.811$ & $>6.56$ & 89.86 & 59.81 & 85.50 & 69.10 & 74.84 \\
ESR $(\mathrm{mm} / \mathrm{h})$ & 0.643 & $0.552-0.727$ & $>44$ & 87.27 & 39.13 & 75.45 & 58.91 & 63.20 \\
RF $(\mathrm{IU} / \mathrm{mL})$ & 0.572 & $0.495-0.646$ & $>101$ & 76.47 & 43.40 & 64.84 & 57.47 & 59.94 \\
Anti-CCP $(\mathrm{U} / \mathrm{mL})$ & 0.544 & $0.455-0.631$ & $>73.2$ & 35.71 & 82.89 & 56.32 & 67.61 & 59.30 \\
\hline
\end{tabular}

Abbreviations: AUC, area under curve; $95 \% \mathrm{Cl}$, 95\% confidence interval; PPV, positive predictive value; NPV, negative predictive value; AC, accuracy; RBC, red blood cells; $\mathrm{Hb}$, hemoglobin; PLT, platelet; RPR, red blood cells-platelet ratio; HPR, hemoglobin-platelet ratio; CRP, C-reactive protein; ESR, erythrocyte sedimentation rate; RF, rheumatoid factor; Anti-CCP, anti-cyclic citrullinated peptide. 

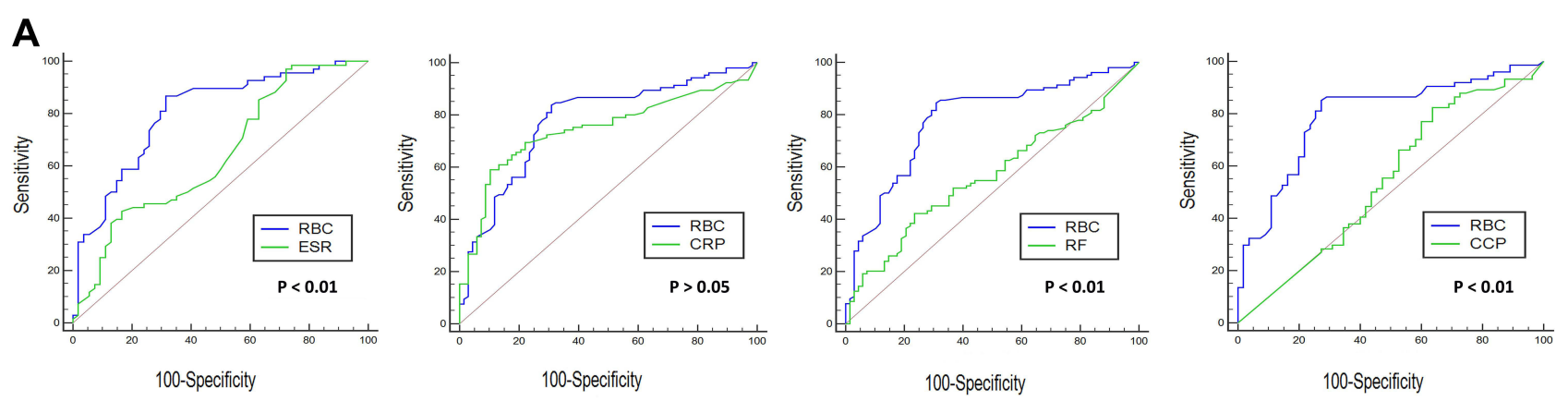

B
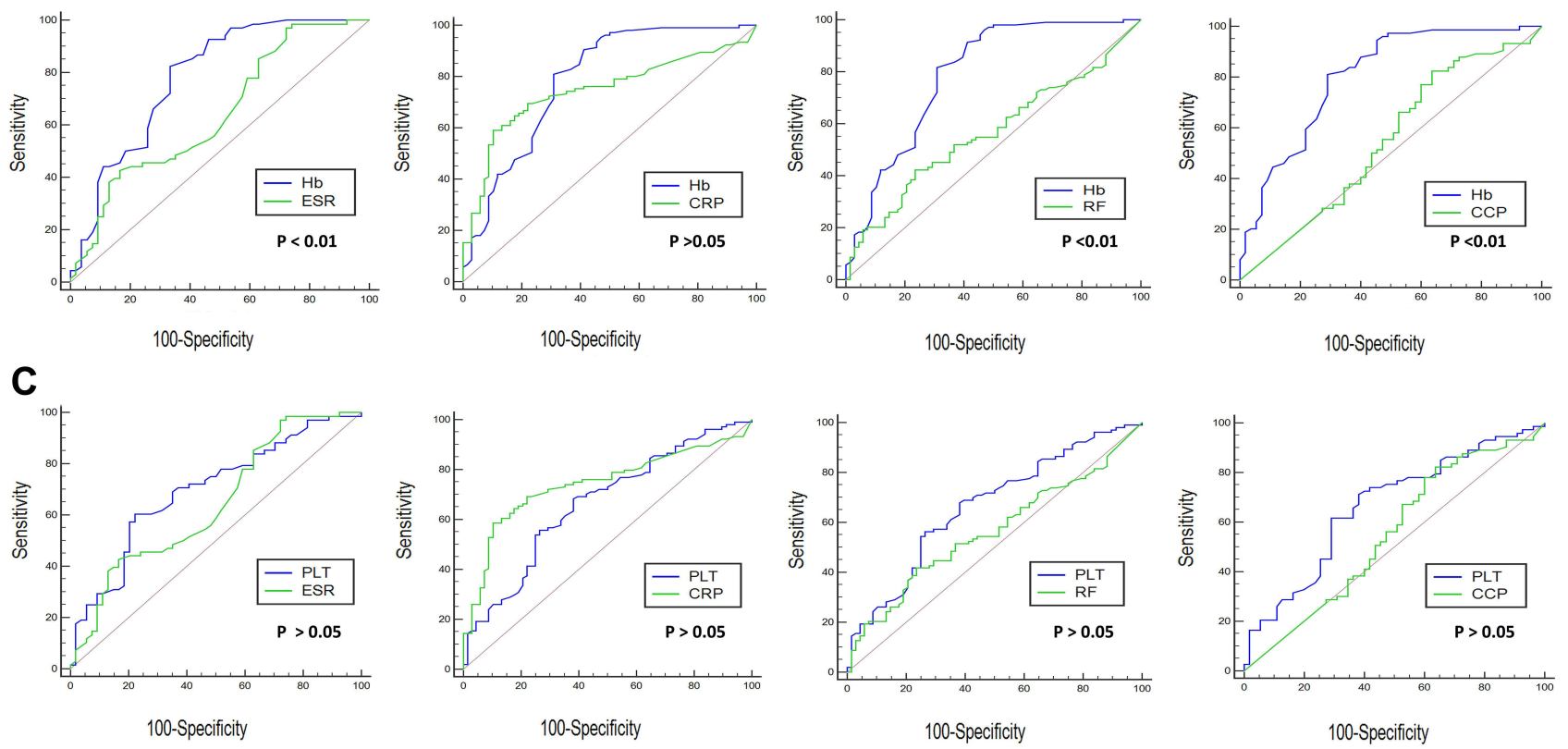

Figure 2 Performance of blood RBC, Hb, and PLT in discriminating active RA from inactive RA. Comparison of ROC curves between RBC (A), Hb (B) or PLT (C) and other parameters including ESR, CRP, RF and Anti-CCP in differentiating active RA from inactive RA.

Abbreviations: ROC, receiver operating characteristic curve; RBC, red blood cells; Hb, hemoglobin; PLT, platelet; ESR, erythrocyte sedimentation rate; CRP, C-reactive protein; RF, rheumatoid factor; Anti-CCP, anti-cyclic citrullinated peptide antibody.

activity of RA patients who were scheduled for blood test, and thus we could find some new indices to identify the disease activity of RA patients. In this study, we confirmed, for the first time, blood PLT and RBC related indices RPR and HPR were significantly associated with the severity of RA.

Recently PLT has been demonstrated to be involved in the inflammatory process of RA. ${ }^{19}$ The number of PLT

Table 5 Correlations of RBC, Hb, PLT, RPR and HPR with Indices of Disease Activity in RA Patients

\begin{tabular}{|c|c|c|c|c|c|c|}
\hline \multirow[t]{2}{*}{ Parameters } & \multicolumn{2}{|c|}{ CRP (mgL) } & \multicolumn{2}{|c|}{$\operatorname{ESR}(\mathrm{mm} / \mathrm{h})$} & \multicolumn{2}{|c|}{ DAS28-CRP } \\
\hline & $\mathbf{r}$ & $P$-value & $\mathbf{r}$ & $P$-value & $\mathbf{r}$ & $P$-value \\
\hline $\operatorname{RBC}\left(10^{12} / \mathrm{L}\right)$ & -0.289 & $<0.001$ & -0.481 & $<0.001$ & -0.428 & $<0.001$ \\
\hline $\mathrm{Hb}(\mathrm{g} / \mathrm{L})$ & $-0.34 I$ & $<0.001$ & -0.569 & $<0.001$ & -0.489 & $<0.001$ \\
\hline $\operatorname{PLT}\left(10^{9} / \mathrm{L}\right)$ & 0.284 & $<0.001$ & 0.331 & $<0.001$ & 0.327 & $<0.001$ \\
\hline RPR & -0.397 & $<0.001$ & -0.329 & $<0.001$ & -0.310 & $<0.001$ \\
\hline HPR & -0.402 & $<0.001$ & -0.362 & $<0.001$ & -0.293 & $<0.001$ \\
\hline
\end{tabular}

Abbreviations: CRP, C-reactive protein; ESR, erythrocyte sedimentation rate; DAS28-CRP, Disease Activity Score in 28 joints based on C-reactive protein; RBC, red blood cells; Hb, hemoglobin; PLT, platelet; RPR, red blood cells-platelet ratio; HPR, hemoglobin-platelet ratio. 
was reported to be significantly higher in RA patients with high disease activity compared to those with low to moderate disease activity. ${ }^{20}$ Moreover, a previous study revealed that mean volume of PLT was significantly associated with RA disease activity. ${ }^{21}$ PLT derived growth factor was indicated to be participated in the invasion of synovial membranes and angiogenesis that are the characteristics of RA. ${ }^{22,23}$ PLT could function as delivering vehicles carrying major amounts of cytokines, chemokines, and growth factors which were important to sustain autoimmune pathways. ${ }^{12,24}$ Notably, a study from KnijffDutmer et al showed that PLT counts were similar in the three groups including active RA, inactive RA and healthy controls. ${ }^{25}$ However, the levels of platelet-derived microparticles (PMPs) in RA patients were evidently higher than those in healthy controls. PMPs were also correlated with disease activity of RA. ${ }^{25}$ Our results showed that there were significant difference in the number of blood PLT between RA patients and the healthy controls. Considering the significance of PMPs in RA inflammation, we will investigate the role of PMPs in RA patients in our further study.

The inflammatory milieu in RA has been demonstrated to modulate erythropoiesis. There are two main reasons responsible for inflammation-induced anemia in RA. Firstly, erythropoietin (EPO) gene transcription could be suppressed by proinflammatory cytokines such as IL-1, IL-6 and TNF- $\alpha .^{26,27}$ Secondly, these proinflammatory cytokines could inhibit the effect of EPO on erythroid progenitors in bone marrow. ${ }^{28}$ RA patients with early stage were reported to present anemia which was caused by IL6-induced suppression of erythropoiesis in bone marrow. ${ }^{29}$ In addition, RBC in RA patients have been shown to be affected by the presence of generated free radicals and excessive amounts of proteins circulating in the blood. ${ }^{30}$ Notably, accelerating evidence revealed that RBC-derived microparticles (RMPs) could directly contribute to the pathogenesis of RA via their regulation on immune cell activation. ${ }^{31}$ In our study, $\mathrm{Hb}$ concentration was decreased in RA patients as compared to the health controls, and the level of $\mathrm{Hb}$ concentration was lower in active RA patients than that in the inactive group. A finding similar to ours was observed in the study by Padjen et $\mathrm{al}^{32}$ who identified that changes in $\mathrm{Hb}$ levels were strongly associated with disease activity of RA patients. Our results demonstrated that blood $\mathrm{Hb}$ concentration was negatively correlated with the indices of RA disease activity such as DAS 28-CRP, CRP and ESR.
The interaction between PLT and RBC bears a helpful understanding of RA pathophysiology, as it has been shown that RMPs were capable of inducing PLT hyperstimulation following collagen activation in an in vitro study. ${ }^{33}$ These RMPs were also confirmed to induce ex vivo PLT-PLT aggregates. Moreover, some adhesion proteins have been identified to be involved in the direct contact between PLT and RBC. For example, as a family member of glycoproteins, adhesion molecule 4 (ICAM-4 or CD242) on RBC membranes could directly bind to the integrin $\alpha \mathrm{IIb} \beta 3$ of PLT, illustrating the direct effect of $\mathrm{RBC}$ on the activation of thrombotic and inflammatory pathways. $^{14,34}$ Given that interaction between PLT and RBC plays a role in the pathogenesis of chronic inflammation of RA, so we evaluated the association of RPR and HPR with the disease activity in RA. Our results showed that RPR and HPR were significantly related to the severity of RA.

However, there were several limitations in this study. Firstly, this study was a retrospective analysis of the data on RA patients, and selection bias cannot be eliminated completely. The controls did not include patients with osteoarthritis or other autoimmune diseases with clinical manifestations similar to RA, such as gout arthritis and so on. Secondly, this study included only 178 patients with RA which were from a single center. Therefore, a multicenter prospective study with a large-scale sample is still required to confirm the accuracy of the results. Additionally, given the effect of disease-modifying antirheumatic drugs (DMARDs) on the disease activity of RA patients, we intend to investigate the association of these blood PLT and RBC related indices with the disease activity in newly diagnosed RA patients who have not received the treatment in our further study.

\section{Conclusions}

In summary, this study systematically investigated the role of PLT, RBC, Hb, RPR and HPR as biomarkers in determining the disease activity of RA. We found that PLT was elevated in RA patients and positively correlated with RA disease activity. RBC, Hb, RPR and HPR were found to be decreased in RA patients and negatively correlated with RA disease activity.

\section{Ethical Approval}

The study was conducted in accordance with the declaration of Helsinki and approved by the Research Committee of Human Investigation of Xi'an Jiaotong University 
Health Science Center and the written informed consent was obtained by all subjects.

\section{Acknowledgment}

We would like to thank Mrs. Junrong Wang for facilitating the data collection.

\section{Funding}

This study was supported by National Natural Science Foundation of China (No. 81802129) and Science and Technology Planning Project of Shaanxi Province of China (No. 2017SF-128 and No. 2020SF-260).

\section{Disclosure}

The authors certify that there is no conflict of interest with any financial organization regarding the material discussed in the manuscript.

\section{References}

1. Smolen JS, Aletaha D, McInnes IB. Rheumatoid arthritis. Lancet. 2016;388(10055):2023-2038. doi:10.1016/S0140-6736(16)30173-8

2. Aletaha D, Smolen JS. Diagnosis and management of rheumatoid arthritis: a review. JAMA. 2018;320(13):1360-1372. doi:10.1001/ jama.2018.13103

3. Kay J, Upchurch KS. ACR/EULAR 2010 rheumatoid arthritis classification criteria. Rheumatology. 2012;51(Suppl 6):vi5-vi9. doi:10.1093/rheumatology/kes279

4. Krabben A, Abhishek A, Britsemmer K, et al. Risk of rheumatoid arthritis development in patients with unclassified arthritis according to the 2010 ACR/EULAR criteria for rheumatoid arthritis. Rheumatology. 2013;52(7):1265-1270. doi:10.1093/rheumatology/ ket011

5. Conigliaro P, Chimenti MS, Triggianese P, et al. Autoantibodies in inflammatory arthritis. Autoimmun Rev. 2016;15(7):673-683. doi:10.1016/j.autrev.2016.03.003

6. Bakogiannis C, Sachse M, Stamatelopoulos K, Stellos K. Plateletderived chemokines in inflammation and atherosclerosis. Cytokine. 2019;122:154157. doi:10.1016/j.cyto.2017.09.013

7. Schulz C, Schafer A, Stolla M, et al. Chemokine fractalkine mediates leukocyte recruitment to inflammatory endothelial cells in flowing whole blood: a critical role for P-selectin expressed on activated platelets. Circulation. 2007;116(7):764-773. doi:10.1161/ CIRCULATIONAHA.107.695189

8. Koupenova M, Clancy L, Corkrey HA, Freedman JE. Circulating platelets as mediators of immunity, inflammation, and thrombosis. Circ Res. 2018;122(2):337-351. doi:10.1161/CIRCRESAHA.1 17.310795

9. Zandman-Goddard G, Shoenfeld Y. Ferritin in autoimmune diseases. Autoimmun Rev. 2007;6(7):457-463.

10. McDevitt MA, Xie J, Gordeuk V, Bucala R. The anemia of malaria infection: role of inflammatory cytokines. Curr Hematol Rep. 2004;3 (2):97-106.

11. Thawani N, Tam M, Stevenson MM. STAT6-mediated suppression of erythropoiesis in an experimental model of malarial anemia. Haematologica. 2009;94(2):195-204. doi:10.3324/haematol.13422

12. Habets KL, Huizinga TW, Toes RE. Platelets and autoimmunity. Eur J Clin Invest. 2013;43(7):746-757. doi:10.1111/eci.12101
13. Xu T, Zhang G, Lin $\mathrm{H}$, et al. Clinical characteristics and risk factors of diffuse alveolar hemorrhage in systemic lupus erythematosus: a systematic review and meta-analysis based on observational studies. Clin Rev Allergy Immunol. 2020;59:295-303.

14. Du VX, Huskens D, Maas C, Al Dieri R, de Groot PG, de Laat B. New insights into the role of erythrocytes in thrombus formation. Semin Thromb Hemost. 2014;40(1):72-80. doi:10.1055/s-00331363470

15. Wang H, Wang J, Huang R, et al. Red blood cell distribution width for predicting significant liver inflammation in patients with autoimmune hepatitis. Eur $J$ Gastroenterol Hepatol. 2019;31 (12):1527-1532. doi:10.1097/MEG.0000000000001447

16. Aletaha D, Neogi T, Silman AJ, et al. 2010 rheumatoid arthritis classification criteria: an American College of Rheumatology/ European League Against Rheumatism collaborative initiative. Ann Rheum Dis. 2010;69(9):1580-1588. doi:10.1136/ard.2010.138461

17. Inoue E, Yamanaka H, Hara M, Tomatsu T, Kamatani N. Comparison of Disease Activity Score (DAS)28- erythrocyte sedimentation rate and DAS28- C-reactive protein threshold values. Ann Rheum Dis. 2007;66(3):407-409. doi:10.1136/ard.2006.054205

18. Nakano S, Morimoto S, Suzuki S, et al. Immunoregulatory role of IL-35 in T cells of patients with rheumatoid arthritis. Rheumatology. 2015;54(8):1498-1506. doi:10.1093/rheumatology/keu528

19. Olumuyiwa-Akeredolu OO, Page MJ, Soma P, Pretorius E. Platelets: emerging facilitators of cellular crosstalk in rheumatoid arthritis. Nat Rev Rheumatol. 2019;15(4):237-248. doi:10.1038/s41584-019-0187-9

20. Talukdar M, Barui G, Adhikari A, Karmakar R, Ghosh UC, Das TK. A study on association between common haematological parameters and disease activity in rheumatoid arthritis. J Clin Diagn Res. 2017;11(1):EC01-EC04. doi:10.7860/JCDR/2017/23524.9130

21. Tekeoglu I, Gurol G, Harman H, Karakece E, Ciftci IH. Overlooked hematological markers of disease activity in rheumatoid arthritis. Int $J$ Rheum Dis. 2016;19(11):1078-1082. doi:10.1111/1756$185 X .12805$

22. Rice JW, Veal JM, Fadden RP, et al. Small molecule inhibitors of Hsp90 potently affect inflammatory disease pathways and exhibit activity in models of rheumatoid arthritis. Arthritis Rheum. 2008;58 (12):3765-3775. doi:10.1002/art.24047

23. Charbonneau M, Lavoie RR, Lauzier A, Harper K, McDonald PP, Dubois CM. Platelet-derived growth factor receptor activation promotes the prodestructive invadosome-forming phenotype of synoviocytes from patients with rheumatoid arthritis. J Immunol. 2016;196 (8):3264-3275. doi:10.4049/jimmunol.1500502

24. Boilard E, Blanco P, Nigrovic PA. Platelets: active players in the pathogenesis of arthritis and SLE. Nat Rev Rheumatol. 2012;8 (9):534-542. doi:10.1038/nrrheum.2012.118

25. Knijff-Dutmer EA, Koerts J, Nieuwland R, Kalsbeek-Batenburg EM, van de Laar MA. Elevated levels of platelet microparticles are associated with disease activity in rheumatoid arthritis. Arthritis Rheum. 2002;46(6):1498-1503. doi:10.1002/art.10312

26. Ferrucci L, Guralnik JM, Woodman RC, et al. Proinflammatory state and circulating erythropoietin in persons with and without anemia. Am J Med. 2005;118(11):1288. doi:10.1016/j.amjmed.2005.06.039

27. La Ferla K, Reimann C, Jelkmann W, Hellwig-Burgel T. Inhibition of erythropoietin gene expression signaling involves the transcription factors GATA-2 and NF-kappaB. FASEB J. 2002;16(13):1811-1813. doi:10.1096/fj.02-0168fje

28. Grigorakaki C, Morceau F, Chateauvieux S, Dicato M, Diederich M. Tumor necrosis factor alpha-mediated inhibition of erythropoiesis involves GATA-1/GATA-2 balance impairment and PU.1 over-expression. Biochem Pharmacol. 2011;82(2):156-166. doi:10.1016/j.bcp.2011.03.030

29. Nikolaisen C, Figenschau Y, Nossent JC. Anemia in early rheumatoid arthritis is associated with interleukin 6-mediated bone marrow suppression, but has no effect on disease course or mortality. J Rheumatol. 2008;35(3):380-386. 
30. Staron A, Makosa G, Koter-Michalak M. Oxidative stress in erythrocytes from patients with rheumatoid arthritis. Rheumatol Int. 2012;32 (2):331-334. doi:10.1007/s00296-010-1611-2

31. Olumuyiwa-Akeredolu OO, Pretorius E. Platelet and red blood cell interactions and their role in rheumatoid arthritis. Rheumatol Int 2015;35(12):1955-1964. doi:10.1007/s00296-015-3300-7

32. Padjen I, Ohler L, Studenic P, Woodworth T, Smolen J, Aletaha D. Clinical meaning and implications of serum hemoglobin levels in patients with rheumatoid arthritis. Semin Arthritis Rheum. 2017;47 (2):193-198. doi:10.1016/j.semarthrit.2017.03.001
33. Valles J, Santos MT, Aznar J, et al. Erythrocytes metabolically enhance collagen-induced platelet responsiveness via increased thromboxane production, adenosine diphosphate release, and recruitment. Blood. 1991;78(1):154-162. doi:10.1182/blood.V78.1.154.154

34. Hermand P, Gane P, Huet M, et al. Red cell ICAM-4 is a novel ligand for platelet-activated alpha IIbbeta 3 integrin. J Biol Chem. 2003;278 (7):4892-4898. doi:10.1074/jbc.M211282200
International Journal of General Medicine

\section{Publish your work in this journal}

The International Journal of General Medicine is an international, peer-reviewed open-access journal that focuses on general and internal medicine, pathogenesis, epidemiology, diagnosis, monitoring and treatment protocols. The journal is characterized by the rapid reporting of reviews, original research and clinical studies across all disease areas. The manuscript management system is completely online and includes a very quick and fair peer-review system, which is all easy to use. Visit http://www.dovepress.com/ testimonials.php to read real quotes from published authors. 